\title{
Magnetically Triggered Release of Entrapped Bioactive Proteins from Thermally Responsive Polymer-Coated Iron Oxide Nanoparticles for Stem-Cell Proliferation
}

\author{
Matthew Walker, Iain Will, Andrew Pratt,* Victor Chechik,* Paul Genever,* and Daniel Ungar*
}

Cite This: ACS Appl. Nano Mater. 2020, 3, 5008-5013

Read Online

ACCESS

Џlll Metrics \& More

回国 Article Recommendations

Supporting Information

ABSTRACT: Nanoparticles could conceal bioactive proteins during therapeutic delivery, avoiding side effects. Superparamagnetic iron oxide nanoparticles (SPIONs) coated with a temperature-sensitive polymer were tested for protein release. We show that coated SPIONs can entrap test proteins and release them in a temperature-controlled manner in a biological system. Magnetically heating SPIONs triggered protein release at bulk solution temperatures below the polymer transition. The entrapped growth factor Wnt3a was inactive until magnetically triggered release, upon which it could increase mesenchymal stem cell proliferation. Once the polymer transition will be chemically adjusted above body temperature, this system could be used for targeted cell stimulation in model animals and humans.

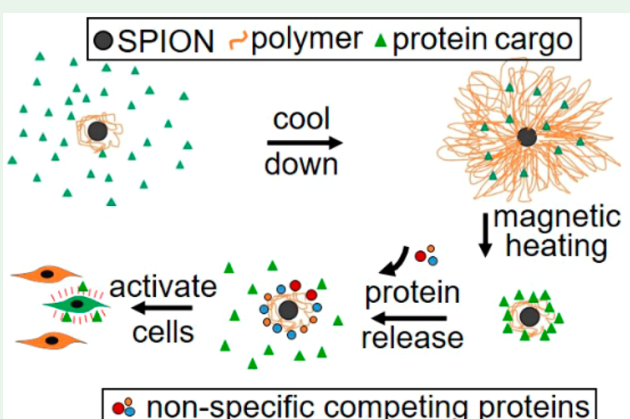

KEYWORDS: superparamagnetic iron oxide nanoparticles, mesenchymal stem cells, in vivo protein delivery, temperature-sensitive polymer, magnetic protein release, polymer-coated nanoparticles

$\mathrm{D}$ eveloping controlled release for bioactive substances has revolutionized many bioscience and medical applications. For example, photoactivation of caged small-molecule drugs has been instrumental for the detailed investigation of biological processes and drug mechanisms. ${ }^{1}$ Moreover, photoactivatable drugs have found use in medical applications in which the timing and location of drug action need careful control. $^{2}$ Examples of caged photoactivatable proteins have been developed, but this requires chemical protein modifications and is unavailable for many protein types and applications. ${ }^{3}$ A generic controlled protein delivery system would, however, be of great importance to a range of medical applications. For example, the Wnt3a protein can stimulate osteoblast progenitor pool proliferation and thereby promote bone formation to assist chronic fracture healing. ${ }^{4}$ Yet systemic Wnt3a administration would raise safety concerns due to the role of canonical Wnt $/ \beta$-catenin signaling in tumor formation. ${ }^{5}$ Hence, concealed delivery and controlled release at the target will be needed for therapeutic use of Wnt3a as well as many other potentially therapeutic proteins. A more versatile alternative to photoactivation could be the magnetic release of proteins caged into nanoparticles.

Temperature-sensitive polymers have in the past been used as scaffolds to release bioactive compounds following implantation because of their ability to undergo a fully reversible gel-to-liquid phase transition. ${ }^{6}$ Phase-transition temperatures can be tuned between 30 and $45^{\circ} \mathrm{C}$ by alteration of the monomer structure or by copolymerization. ${ }^{7}$ Most commonly used is poly $(N$-isopropylacrylamide) (PNIPAM), which has a lower critical solution temperature (LCST) of 32 ${ }^{\circ} \mathrm{C}$, above which the polymer expels water, going from a swollen hydrated state to a shrunken hydrophobic state. ${ }^{6}$ Vascular endothelial growth factor (VEGF) could, for example, be released from a PNIPAM scaffold containing dispersed superparamagnetic iron oxide nanoparticles (SPIONs), which were used to contract the gel by heating. Expelled VEGF was able to promote human umbilical vein endothelial cell growth in a culture. ${ }^{8}$

A more precise tool for the delivery of bioactives are nanoparticles. These can distribute throughout the body, while concealing bioactive proteins during transit if fitted with a trigger mechanism to enable controlled release at the target site. ${ }^{9}$ Using a superparamagnetic core (e.g., SPIONs) enables controlled release of bioactive compounds through magnetic heating, as shown for VEGF. ${ }^{8}$ SPIONs are made up of a magnetite/maghemite core, generally 5-150 nm in size. They had previously been developed for magnetic hyperthermia, imaging, and cell-tracking applications. ${ }^{10,11}$ Moreover, SPIONs functionalized with thermally responsive polymers have been demonstrated to release small-molecule drugs, such as

Received: May 4, 2020

Accepted: May 15, 2020

Published: May 15, 2020 
a)

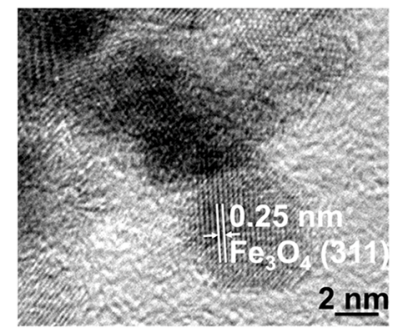

b)

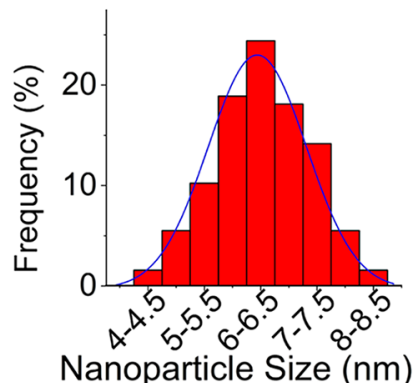

c)

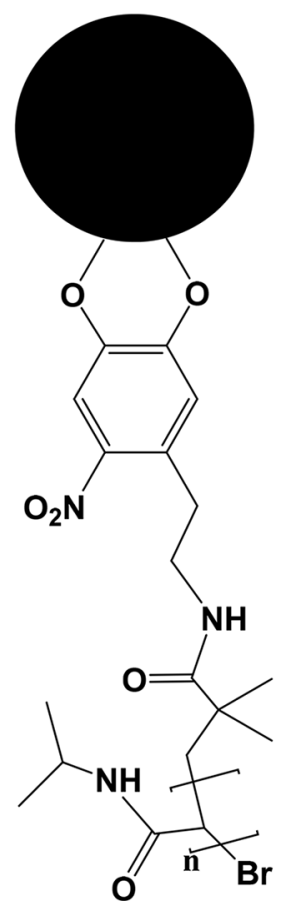

d)

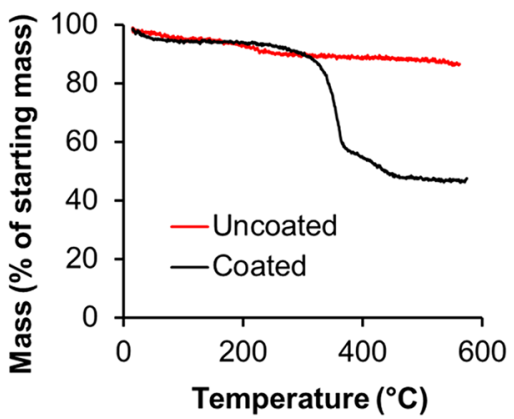

e)
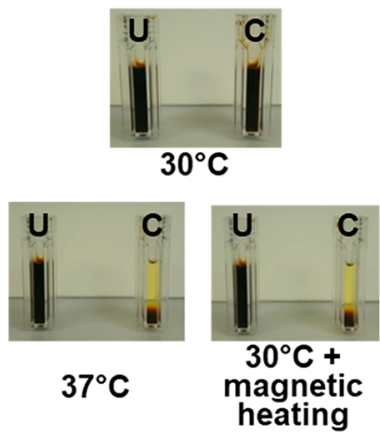

Figure 1. Characterization of polymer-coated SPIONs. (a) High-resolution transmission electron micrograph of nanoparticles and (b) the associated size distribution, as determined from lower-magnification micrographs. For calculation of the shown lattice plane distance, see also Figure S1. A Gaussian fit over the SPION size distribution is shown. ${ }^{19}$ (c) Schematic of nitrodopamine-terminated PNIPAM and its attachment to the SPION surface (the black sphere denotes a SPION). (d) TGA of $10 \mathrm{mg}$ each of uncoated and PNIPAM-coated SPION samples, which were heated under nitrogen gas at a ramp rate of $10{ }^{\circ} \mathrm{C} / \mathrm{min}$ between 0 and $600{ }^{\circ} \mathrm{C}$. (e) Photographs of $10 \mathrm{mg} / \mathrm{mL}$ PNIPAM-coated (C) and uncoated (U) SPIONs incubated at the indicated temperatures for $30 \mathrm{~min}$ in plastic cuvettes. For magnetic heating, the sample was exposed to a $108 \mathrm{kHz} /$ $0.67 \mathrm{~T}$ alternating magnetic field before quick transfer to the cuvette for photographing.

doxorubicin, upon magnetic-field-induced heating. ${ }^{12}$ The interactions between proteins and the PNIPAM shell surrounding the SPIONs, however, are more complex, and reports are often contradictory. Protein-PNIPAM interactions are likely to be strongly dependent on the polymer density and morphology in the shell. ${ }^{13}$ The release of a protein from a polymer-coated nanoparticle is therefore more complex than simply squeezing out the protein in the aqueous phase during hydrophobic collapse, as was observed for VEGF in a SPIONdoped hydrogel. ${ }^{8}$ In many cases, adsorption of proteins on PNIPAM substrates was observed above the LCST, presumably driven by hydrophobic interactions that could hinder protein release in the collapsed state. ${ }^{14}$

In this study, we provide proof-of-concept for the development of SPIONs coated with a temperature-sensitive polymer, which could entrap proteins below the polymer's LCST of 32 ${ }^{\circ} \mathrm{C}$, concealing their bioactivity. Subsequent magnetic heating triggered collapse of the polymer shell at bulk temperatures well below the LCST. Although the collapsed polymer retained the cargo protein through hydrophobic interactions, nonspecific competing proteins promoted the release of cargo proteins, which, in turn, could perform bioactive functions, such as enhancing stem cell proliferation, in a model system. We propose from these results that SPIONs, once coated with polymers transitioning above $42{ }^{\circ} \mathrm{C}$ and equipped with the means for targeting, could provide an effective controlled in vivo delivery system for bioactive proteins.

Water-soluble thermally responsive SPIONs with an average size of $6.3 \pm 0.9 \mathrm{~nm}$, which can entrap and then release bioactive proteins, were synthesized using a modified polyol process (Figure 1a,b). ${ }^{15}$ The measured lattice spacing of 0.25 $\mathrm{nm}$ (Figures $1 \mathrm{la}$ and S1) is in good agreement with $\mathrm{Fe}_{3} \mathrm{O}_{4},{ }^{16}$ as were X-ray photoelectron spectroscopy measurements ${ }^{17}$ (Figure S2). Vibrating-sample magnetometry confirmed superparamagnetism (Figure S3), ${ }^{18}$ while a specific absorption rate of $3.6 \mathrm{~W} / \mathrm{g}$ of $\mathrm{Fe}$ was determined in water by measuring a heating rate of $0.26{ }^{\circ} \mathrm{C} / \mathrm{min}$ at $5 \mathrm{mg} / \mathrm{mL}$ SPION concentration upon exposure to a $0.67 \mathrm{~T} / 108 \mathrm{kHz}$ alternating magnetic field. Given their small size $(<12 \mathrm{~nm})$, Néel relaxation will dominate heating, while the contribution from mechanical movement (Brownian relaxation) will be negligible. ${ }^{19,20}$

A thermally responsive coating was achieved using PNIPAM, which has well-documented phase-transition properties, and has been widely used in biological applications, including SPION coating. ${ }^{8,12}$ Using an established atomtransfer-radical polymerization method, PNIPAM polymers (Figure S4a) with a number-average molecular weight $\left(M_{n}\right)$ of $12.99 \mathrm{kDa}$ (Figure S4b) were synthesized. The addition of a terminal nitrocatechol group enabled SPION coating (Figures $1 \mathrm{c}, \mathrm{S5}$, and S6). ${ }^{21}$ Thermogravimetric analysis (TGA) showed a total mass loss of $50.3 \%$ when the organic polymer shell was decomposed above $200{ }^{\circ} \mathrm{C}$ (Figure 1d). This equates to a grafting density of 0.26 chains $/ \mathrm{nm}^{2}$ or 32 polymer chains per nanoparticle, consistent with published data. ${ }^{21}$ PNIPAMcoated SPIONs remained readily dispersed in water below the polymer's LCST (Figure 1e, top). Coated SPIONs precipitated due to aggregation when incubated above the LCST or when heated with an alternating magnetic field (Figure 1e, bottom). We next explored the potential application of these coated SPIONs to entrap and release proteins. 


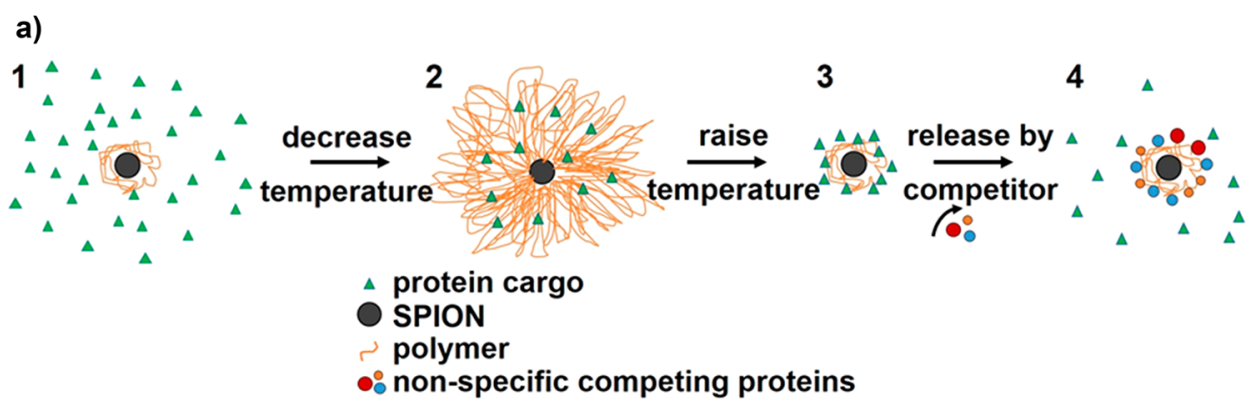

b)
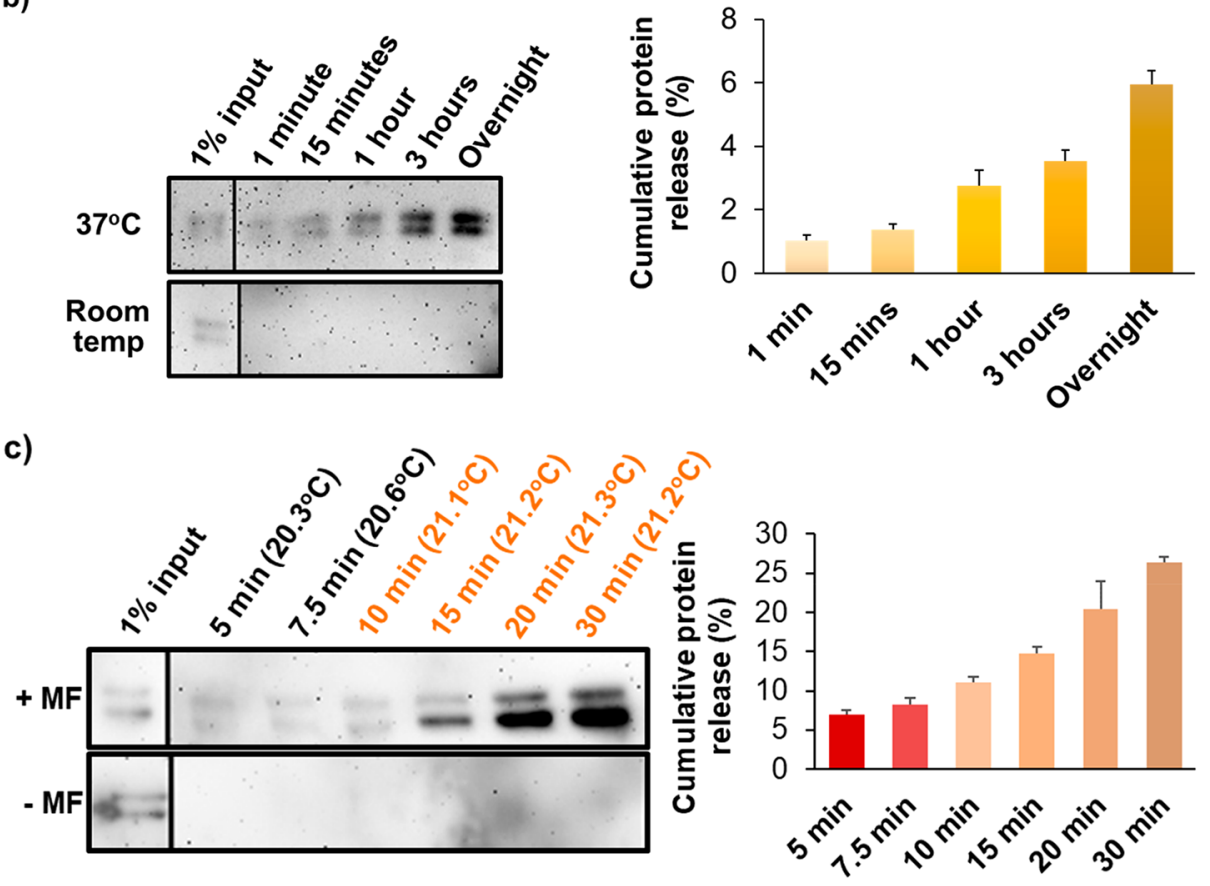

Figure 2. Protein release from PNIPAM-coated SPIONs. (a) Schematic diagram of protein entrapment and release by PNIPAM-coated SPIONs. (1) $1 \mu \mathrm{g}$ of protein was mixed with $1 \mathrm{mg}$ of coated SPIONs above the LCST. Polymer chains were collapsed around the nanoparticle core, and agitation was used to avoid particle aggregation. (2) Upon cooling below the LCST, the polymer shell expanded, and some protein molecules were engulfed by the shell; we call this the entrapping state of the coated SPION. (3) Following removal of excess protein by washes in the presence of competing nonspecific proteins, entrapped proteins could be discharged by polymer collapse above the LCST, (4) which permitted nonspecific competitor proteins to replace the weakly bound cargo proteins, thereby releasing them. (b) (Left) Western blot analysis of the apotransferrin collected from the supernatant, following incubation of apotransferrin-loaded PNIPAM-coated nanoparticles $(1 \mathrm{mg})$ in the presence of $10 \mathrm{mg} / \mathrm{mL}$ RNase B (the nonspecific competitor) at $\mathrm{pH}$ 7.5. SPIONs were briefly collected on one side of the tube with a permanent magnet when the solution was sampled at the indicated time points. (Right) Densitometry of the apotransferrin immunoblot signal used to quantify the amount of apotransferrin released. Error bars denote standard deviation, $n=3.100 \%$ is the amount of protein used for entrapment, given that the amount of protein detected in the washing steps prior to release was negligible. (c) As in part b, but with or without application of an alternating magnetic field $( \pm \mathrm{MF}$ as indicated) turned on constantly for $10 \mathrm{~min}$ and then in pulses of $10 \mathrm{~s}$ on and $10 \mathrm{~s}$ off. The bulk solution temperatures measured during magnetic heating using an IR thermocouple probe are shown in parentheses above each time point. The sample without magnetic heating was maintained at $21{ }^{\circ} \mathrm{C}$. Error bars denote standard deviation, $n=3$. Input lanes are from the same blot images but had to be moved because these were not in lanes adjacent to the release samples. Therefore, a divider line was introduced. Note that the double band of apotransferrin represents differently glycosylated forms whose ratios are batch-dependent.

Entrapment and release of small-molecule cargo from PNIPAM-coated nanoparticles has been reported ${ }^{7,12}$ but not protein entrapment and release. Apotransferrin, a major serum glycoprotein, was used as a model to assess protein entrapment and release. Apotransferrin loading was achieved by first incubating coated SPIONs with the protein above the polymer LCST $\left(37^{\circ} \mathrm{C}\right)$, followed by cooling below the LCST to promote polymer expansion and protein entrapment in the polymer shell (Figure 2a). Shaking above the LCST prevented aggregation-mediated precipitation. Most attempts to release proteins failed even above the LCST (Figure S9a), except treatment at high $\mathrm{pH}$ (data not shown), indicating that proteins are entrapped but strongly associate with the polymer itself.

We found that the incubation of loaded SPIONs in the presence of a competing nonspecific protein $(10 \mathrm{mg} / \mathrm{mL}$ RNase B) at the physiological $\mathrm{pH}$ of 7.4 facilitated apotransferrin release, but only above the LCST (Figure 2b). Thus, in the presence of a competitor, cargo protein can be released in a temperature-controlled manner under physiological conditions (Figure 2a). Note that this competitor effect is not specific to RNase B, as shown for Wnt3a release. This indicates that the presence of competing proteins in tissues at physiological concentrations could enable release of the 
a)

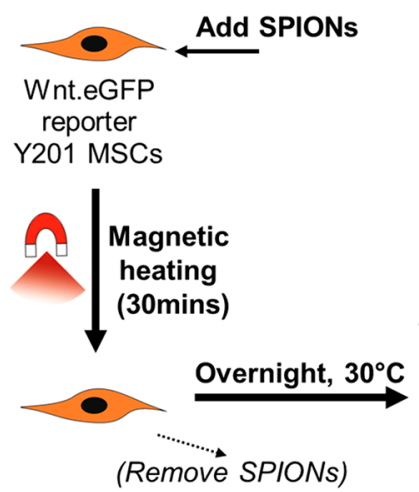

c)

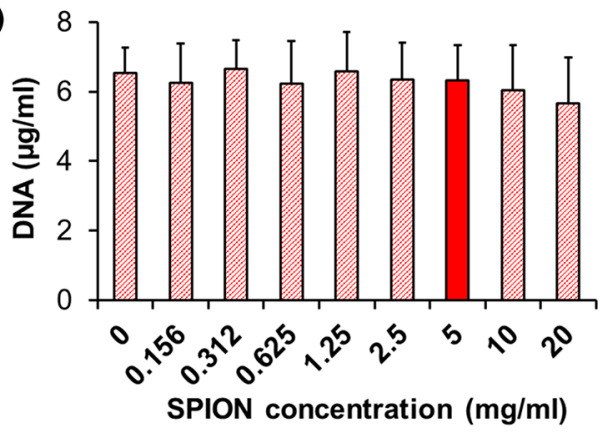

eGFP

measured by

flow cytometry

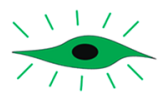

11

(Remove SPIONs) b)

d)
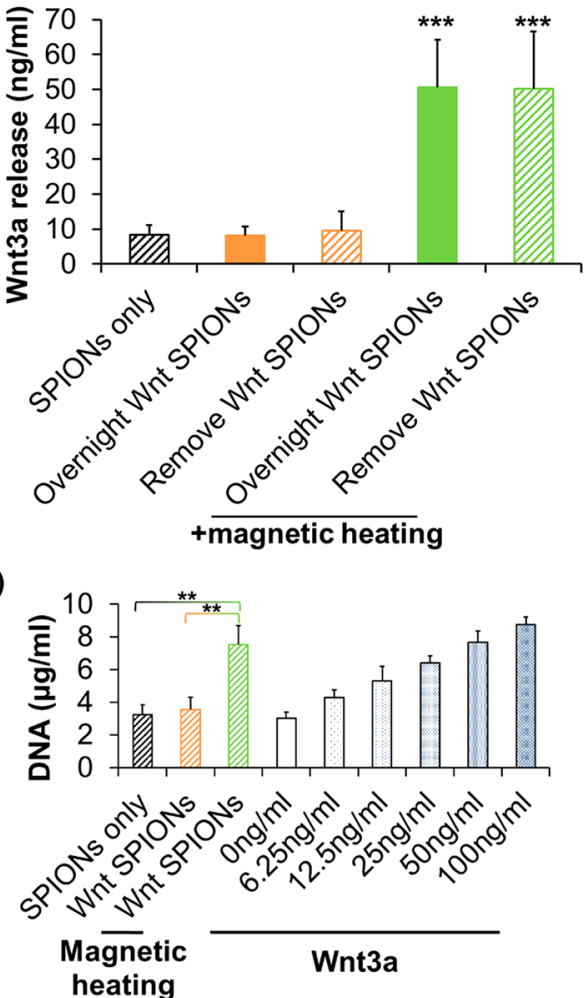

Figure 3. Magnetically activated nanoparticles can release bioactive Wnt3a. (a) Experimental strategy for Wnt-reporter experiments. (b) Quantification of Wnt3a released from $1 \mathrm{mg}$ of SPIONs (loaded with $1 \mu \mathrm{g}$ of Wnt3a), calculated from scatter plots of the Wnt-reporter response measured by flow cytometry (Figure S11c). Flow cytometry was performed following overnight incubation of the Y201 Wnt-reporter MSCs at 30 ${ }^{\circ} \mathrm{C}$. Wnt3a release from SPIONs was triggered for $30 \mathrm{~min}$ in custom-built cell-growth vessels by magnetic treatment that shifted the bulk solution temperature from 21.0 to $24.5{ }^{\circ} \mathrm{C}$. SPIONs were removed following magnetic treatment where indicated. The amount of released Wnt3a was calculated from a calibration curve (Figure S11a,b). Error bars denote standard deviation, $n=4$. *** indicates $p<0.001$ compared with "Overnight Wnt SPIONs" at $30^{\circ} \mathrm{C}$ using Dunnet's multiple comparisons ANOVA. (c) Picogreen staining-based DNA quantification of Y201 MSCs that were incubated for 7 days with protein-free coated SPIONs at the indicated concentrations. Error bars denote standard deviation, $n=3$. The solid red bar shows the SPION concentration used in Wnt3a release experiments. (d) Y201 MSCs treated for 30 min with Wnt3a-loaded (Wnt SPIONs; 1 $\mathrm{mg}$ of SPIONs and $1 \mu \mathrm{g}$ of Wnt3a used in the loading reaction) or protein-free (SPIONs only; $1 \mathrm{mg}$ ) coated SPIONs in the presence of an alternating magnetic field where indicated. At the end of the $30 \mathrm{~min}$ incubation, all SPIONs were removed with a permanent magnet. Cells were then incubated for 5 days at $37{ }^{\circ} \mathrm{C}$, followed by picogreen staining-based DNA quantification. The results for cells incubated with increasing concentrations of Wnt3a only are shown for comparison. Error bars denote standard deviation, $n=3$. ** indicates $p<0.01$ using Tukey's multiple comparisons ANOVA. Application of the alternating magnetic field caused a bulk solution temperature shift of $20.9-24.1^{\circ} \mathrm{C}$. DNA quantification with picogreen staining was based on calibration curves obtained using salmon sperm DNA.

entrapped proteins. We believe that this is the first report of the temperature-controlled release of an entrapped protein from polymer-coated nanoparticles under physiological conditions. We would argue that the interactions between the protein and the hydrophilic polymer below its LCST are dominated by hydrogen bonding. Steric entanglement of the protein in the polymer chains likely contributes to entrapment (Figure 2a). In contrast, when the polymer collapses into a hydrophobic state, steric entrapment ceases. At the same time, the polymer-protein interactions shift from hydrogen bonding to weaker hydrophobic interactions. The effect of competing proteins suggests that the collapsed polymer shell allows for the binding and release of proteins that associate with it via weak noncovalent hydrophobic interactions.

Having observed temperature-controlled release of protein from the coated SPIONs, we next assessed whether release could be triggered by magnetic heating. Using a bespoke magnetic setup (Figures S7 and S8), apotransferrin-loaded SPIONs were incubated in a $0.67 \mathrm{~T} / 108 \mathrm{kHz}$ alternating magnetic field, while monitoring the sample temperature in real time and sampling for released protein. Apotransferrin release was indeed detected when the SPION core was magnetically heated from an ambient temperature of $21{ }^{\circ} \mathrm{C}$ (Figures $2 \mathrm{c}$ and S9b). Interestingly, released apotransferrin was detected at bulk solution temperatures well below the LCST (Figure 2c). It is likely that the thermal energy generated in the SPION core promotes localized polymer collapse during transmission through the shell to the bulk solution, permitting protein release. Importantly, cumulative release of apotransferrin could be achieved without a significant temperature increase in the bulk solution when magnetic heating was applied in short pulses (Figure 2c). This is in agreement with previous reports that an alternating magnetic field can heat the SPION core to a much greater extent than the consequent heating of the bulk solution. ${ }^{22}$ Cumulative release appears to be somewhat more efficient during pulsed magnetic heating. Such release did, however, depend on recurrent SPION heating because further release was halted when magnetic heating was switched off (Figure S9c). This implies that the PNIPAM transition on the particle surface is reversible and the temperature gradient required for polymer collapse quickly dissipates. During magnetic heating experiments, where the 
bulk solution temperature remained below the LCST, we never observed particle aggregation. This is in contrast to reversible aggregation during release experiments using bulk heating (Figure $2 b$ ). In apotransferrin release experiments, $10-25 \%$ of the model protein used for entrapment was recovered. We believe that the remaining protein was still associated with the coated SPIONs because we did not detect signs of protein degradation. To our best knowledge, this is the first report of a magnetically triggered release of proteins entrapped in polymer-coated nanoparticles. We turned to testing how the developed coated SPIONs can shield and release bioactive proteins.

To test bioactive protein release in response to magnetic heating, we used the growth factor Wnt3a, together with an immortalized human mesenchymal stem cell (MSC) line (Y201) engineered to produce enhanced green fluorescent protein (eGFP) in response to Wnt signaling (Figure S1la,b). ${ }^{23}$ Wnt3a released upon magnetic triggering of the coated SPIONs can bind to Wnt receptors on the MSC surface, initiating signaling resulting in eGFP expression to be quantified by flow cytometry (Figure 3a). For compatibility with the magnetic heating setup, we used custom-made tubes that permit normal cell growth (Figure S10). MSCs treated with an alternating magnetic field in the presence of Wnt3aloaded SPIONs, produced increased eGFP expression (Figures $3 \mathrm{~b}$ and S11c). This showed magnetic-heating-induced active Wnt3a release, while the bulk solution remained under 24.5 ${ }^{\circ} \mathrm{C}$, well below the polymer's LCST, and in a safe range to prevent the protein's denaturation (Figure S12). These experiments used competing proteins present in fetal bovine serum, part of the growth medium and mimicking a natural cellular environment.

In some of the above experiments, SPIONs carrying entrapped Wnt3a were removed following the $30 \mathrm{~min}$ magnetic stimulation. In other experiments, the Wnt3a-loaded particles remained in the culture medium following the $30 \mathrm{~min}$ magnetic stimulation for the whole overnight incubation prior to fluorescence analysis. The measured eGFP level was indistinguishable between these conditions, suggesting that active Wnt3a release stopped once magnetic stimulation was halted. Moreover, Wnt3a-loaded SPIONs did not trigger signaling in the absence of magnetic heating, showing that entrapped Wnt3a remained biologically inactive within the polymer shell (Figure $3 \mathrm{~b}$ ). These results show that PNIPAMcoated SPIONs can entrap a protein factor to shield it from its biological target and can release this same protein in a bioactive form upon heating by an alternating magnetic field. This technology could thus find application with biopharmaceuticals that have harmful side effects when acting away from an injury site.

The above experiments demonstrated that Wnt3a released from coated SPIONs was able to activate intracellular signaling and drive transactivation of Wnt target genes. Next, we determined whether the strength of the Wnt signal was sufficient to influence cell function, namely, proliferation, a known biological response of MSCs to Wnt3a exposure (Figure S13). ${ }^{24}$ For these experiments, we used the multipotent clonal human MSC line (Y201), ${ }^{25}$ from which the Wnt eGFP reporter line was generated. In toxicity tests, we could show that SPIONs alone, at concentrations 4 times higher than those used in proliferation experiments, did not affect the viability of Y201 MSCs (Figure 3c). When Wnt3a-loaded SPIONs were added to Y201 MSCs and magnetically stimulated for $30 \mathrm{~min}$ before their removal, the treated cells showed significantly $(p<0.01)$ increased proliferation compared to untreated controls after 5 days (Figure 3d). Thus, the bioactive Wnt3a released from SPIONs by an alternating magnetic field could influence complex cellular behavior. This shows that these coated nanoparticle carriers are capable of influencing important physiological cell responses by controlled release of a bioactive protein factor through remote magnetic heating.

SPIONs are a versatile tool for noninvasive nanomedicinebased therapeutic strategies because of the ease with which they penetrate tissues ${ }^{26}$ and can be triggered with an alternating magnetic field. ${ }^{27}$ Previous applications of SPIONs have included magnetic hyperthermia ${ }^{10}$ and the magnetically driven delivery of small-molecule drugs. ${ }^{7,12}$ Here, we show that SPIONs, when coated with a thermally sensitive polymer shell, can be used to deliver protein growth factors to target cells under physiological conditions in vitro. Exposure of SPIONs to an oscillating magnetic field changed the mode of binding of entrapped proteins. Using two very different model cargo proteins, we showed that, although these proteins had remained bound to the SPIONs, they were released into solution in the presence of nonspecifically binding competing proteins that are naturally present in human tissues. Importantly, release occurred following local heating of the SPION shell, while the bulk solution temperature remains below the LCST. While the protein remained inactive in the entrapped form, upon release, it could trigger the proliferation of MSCs. This should enable the use of this technology without the harmful side effects of hyperthermia. A primary goal for ongoing and future work is to increase the polymer LCST using established methodology. ${ }^{7}$ Increasing the LCST will be an essential next step to make the coated SPION device usable in animal model studies and ultimately for human use.

\section{ASSOCIATED CONTENT}

\section{Supporting Information}

The Supporting Information is available free of charge at https://pubs.acs.org/doi/10.1021/acsanm.0c01167.

Supplementary methods describing SPION and polymer synthesis, coating, physicochemical characterization, and biochemical and cellular assays, supplementary Figures S1-S13 showing detailed physicochemical characteristics of the SPIONs and the polymer coat, additional protein release data and cellular assay calibration experiments, and supplementary references (PDF)

\section{AUTHOR INFORMATION}

\section{Corresponding Authors}

Andrew Pratt - Department of Physics, University of York, York YO10 5DD, U.K.; Email: andrew.pratt@york.ac.uk

Victor Chechik - Department of Chemistry, University of York, York YO10 5DD, U.K.; Email: victor.chechik@york.ac.uk

Paul Genever - Department of Biology, University of York, York YO10 5DD, U.K.; Email: paul.genever@york.ac.uk

Daniel Ungar - Department of Biology, University of York, York YO10 5DD, U.K.; 이이이.org/0000-0002-9852-6160; Email: dani.ungar@york.ac.uk

\section{Authors}

Matthew Walker - Department of Biology, University of York, York YO10 5DD, U.K. 
Iain Will - Department of Electronic Engineering, University of York, York YO10 5DD, U.K.

Complete contact information is available at:

https://pubs.acs.org/10.1021/acsanm.0c01167

\section{Notes}

The authors declare no competing financial interest.

\section{ACKNOWLEDGMENTS}

The authors thank Mark Bentley (Mechanical Workshop, University of York, York, U.K.) for the design of custom-made vessels for cell magnetic heating experiments. The authors acknowledge the EPSRC (strategic studentship EP/M508196/ 1 and impact accelerator funding EP/K504014/1) for funding this work and members of the Cell Biology Laboratory and the Chechik and Pratt groups (University of York, York, U.K.) for the help and guidance provided.

\section{REFERENCES}

(1) Nakagawa, H. Photo-Controlled Release of Small Signaling Molecules to Induce Biological Responses. Chem. Rec. 2018, 18 (12), $1708-1716$

(2) Dorman, G.; Prestwich, G. D. Using photolabile ligands in drug discovery and development. Trends Biotechnol. 2000, 18 (2), 64-77.

(3) Ankenbruck, N.; Courtney, T.; Naro, Y.; Deiters, A. Optochemical Control of Biological Processes in Cells and Animals. Angew. Chem., Int. Ed. 2018, 57 (11), 2768-2798.

(4) Shen, J.; Chen, X. P.; Jia, H. C.; Meyers, C. A.; Shrestha, S.; Asatrian, G.; Ding, C.; Tsuei, R.; Zhang, X. L.; Peault, B.; Ting, K.; Soo, C.; James, A. W. Effects of Wnt3A and Wnt16 on the Osteogenic and Adipogenic Differentiation of Perivascular Stem/Stromal Cells. Tissue Eng., Part A 2018, 24 (1-2), 68-80.

(5) Cook, D. A.; Fellgett, S. W.; Pownall, M. E.; O'Shea, P. J.; Genever, P. G. Wnt-dependent osteogenic commitment of bone marrow stromal cells using a novel GSK3beta inhibitor. Stem Cell Res. 2014, 12 (2), 415-427.

(6) Khutoryanskiy, V. V. e.; Georgiou, T. K. e. TemperatureResponsive Polymers: Chemistry, Properties, and Applications, 1st ed.; Wiley, 2018; p 408.

(7) Li, J. B.; Qu, Y.; Ren, J.; Yuan, W. Z.; Shi, D. L. Magnetocaloric effect in magnetothermally-responsive nanocarriers for hyperthermiatriggered drug release. Nanotechnology 2012, 23 (50), 505706.

(8) Dionigi, C.; Lungaro, L.; Goranov, V.; Riminucci, A.; PineiroRedondo, Y.; Banobre-Lopez, M.; Rivas, J.; Dediu, V. Smart magnetic poly $(\mathrm{N}$-isopropylacrylamide) to control the release of bio-active molecules. J. Mater. Sci.: Mater. Med. 2014, 25 (10), 2365-2371.

(9) Liu, J. F.; Jang, B.; Issadore, D.; Tsourkas, A. Use of magnetic fields and nanoparticles to trigger drug release and improve tumor targeting. Wiley Interdiscip. Rev.: Nanomed. Nanobiotechnol. 2019, 11, 1571.

(10) Banobre-Lopez, M.; Teijeiro, A.; Rivas, J. Magnetic nanoparticle-based hyperthermia for cancer treatment. Rep. Pract Oncol Radiother 2013, 18 (6), 397-400.

(11) Dulinska-Litewka, J.; Lazarczyk, A.; Halubiec, P.; Szafranski, O.; Karnas, K.; Karewicz, A. Superparamagnetic Iron Oxide Nanoparticles-Current and Prospective Medical Applications. Materials 2019, 12 (4), 617.

(12) Purushotham, S.; Chang, P. E. J.; Rumpel, H.; Kee, I. H. C.; Ng, R. T. H.; Chow, P. K. H.; Tan, C. K.; Ramanujan, R. V. Thermoresponsive core-shell magnetic nanoparticles for combined modalities of cancer therapy. Nanotechnology 2009, 20 (30), 305101.

(13) Cole, M. A.; Voelcker, N. H.; Thissen, H.; Horn, R. G.; Griesser, H. J. Colloid probe AFM study of thermal collapse and protein interactions of poly(N-isopropylacrylamide) coatings. Soft Matter 2010, 6 (12), 2657-2667.
(14) Zhang, Y.; Cai, J. Z.; Li, C. H.; Wei, J. Y.; Liu, Z. H.; Xue, W. Effects of thermosensitive poly-(N-isopropylacrylamide) on blood coagulation. J. Mater. Chem. B 2016, 4 (21), 3733-3749.

(15) Carroll, K. J.; Shultz, M. D.; Fatouros, P. P.; Carpenter, E. E. High magnetization aqueous ferrofluid: A simple one-pot synthesis. J. Appl. Phys. 2010, 107 (9), 09B304.

(16) Zhou, G. M.; Wang, D. W.; Li, F.; Zhang, L. L.; Li, N.; Wu, Z. S.; Wen, L.; Lu, G. Q.; Cheng, H. M. Graphene-Wrapped Fe3O4 Anode Material with Improved Reversible Capacity and Cyclic Stability for Lithium Ion Batteries. Chem. Mater. 2010, 22 (18), 5306-5313.

(17) Parkinson, G. S. Iron oxide surfaces. Surf. Sci. Rep. 2016, 71 (1), $272-365$.

(18) Teja, A. S.; Koh, P. Y. Synthesis, properties, and applications of magnetic iron oxide nanoparticles. Prog. Cryst. Growth Charact. Mater. 2009, 55 (1-2), 22-45.

(19) Suto, M.; Hirota, Y.; Mamiya, H.; Fujita, A.; Kasuya, R.; Tohji, K.; Jeyadevan, B. Heat dissipation mechanism of magnetite nanoparticles in magnetic fluid hyperthermia. J. Magn. Magn. Mater. 2009, 321 (10), 1493-1496.

(20) Vallejo-Fernandez, G.; Whear, O.; Roca, A. G.; Hussain, S.; Timmis, J.; Patel, V.; O'Grady, K. Mechanisms of hyperthermia in magnetic nanoparticles. J. Phys. D: Appl. Phys. 2013, 46 (31), 312001.

(21) Kurzhals, S.; Zirbs, R.; Reimhult, E. Synthesis and MagnetoThermal Actuation of Iron Oxide Core-PNIPAM Shell Nanoparticles. ACS Appl. Mater. Interfaces 2015, 7 (34), 19342-19352.

(22) Dong, J. Y.; Zink, J. I. Taking the Temperature of the Interiors of Magnetically Heated Nanoparticles. ACS Nano 2014, 8 (5), 51995207.

(23) Saleh, F.; Carstairs, A.; Etheridge, S. L.; Genever, P. Real-Time Analysis of Endogenous Wnt Signalling in 3D Mesenchymal Stromal Cells. Stem Cells Int. 2016, 2016, 1.

(24) Boland, G. M.; Perkins, G.; Hall, D. J.; Tuan, R. S. Wnt3a promotes proliferation and suppresses osteogenic differentiation of adult human mesenchymal stem cells. J. Cell. Biochem. 2004, 93 (6), $1210-1230$.

(25) James, S.; Fox, J.; Afsari, F.; Lee, J.; Clough, S.; Knight, C.; Ashmore, J.; Ashton, P.; Preham, O.; Hoogduijn, M.; Ponzoni, R. D. A. R.; Hancock, Y.; Coles, M.; Genever, P. Multiparameter Analysis of Human Bone Marrow Stromal Cells Identifies Distinct Immunomodulatory and Differentiation-Competent Subtypes. Stem Cell Rep. 2015, 4 (6), 1004-1015.

(26) Ng, C. P.; Pun, S. H. A perfusable 3D cell-matrix tissue culture chamber for in situ evaluation of nanoparticle vehicle penetration and transport. Biotechnol. Bioeng. 2008, 99 (6), 1490-1501.

(27) Tong, S.; Quinto, C. A.; Zhang, L. L.; Mohindra, P.; Bao, G. Size-Dependent Heating of Magnetic Iron Oxide Nanoparticles. ACS Nano 2017, 11 (7), 6808-6816. 